\title{
STRICTLY CONVEX METRIC SPACES WITH ROUND BALLS AND FIXED POINTS
}

\author{
INESE BULA* \\ Department of Mathematics, University of Latvia \\ Rainis boulv. 19, Rīga, LV-1586, Latvia \\ E-mail: ibula@lanet.lv
}

\begin{abstract}
The paper introduces a notion of strictly convex metric space and strictly convex metric space with round balls. These objects generalize the well known concept of strictly convex Banach space. We prove some fixed point theorems in strictly convex metric spaces with round balls.
\end{abstract}

1. Introduction. Usually we consider convex sets only in a vector space (by Minkowski definition: a closed subset of $\mathbf{R}^{n}$ is convex provided it contains for any two of its points the segment joining them (L. Blumenthal [1], p. 40)). But the notion of "convexity" in a metric space is well-known from work of K. Menger [25]: a metric space $(X, d)$ is said to be (metrically) convex if for any two points $x, y \in X(x \neq y)$ there exists a point $z \in X$ such that $x \neq z \neq y$ and $d(x, y)=d(x, z)+d(z, y)$. From works of K. Menger [25], T. Botts [2], W. L. Klee [21], D. C. Kay and E. W. Womble [17], V. P. Soltan [28], T. H. Kim and W. A. Kirk [18] there are two solid indications:

a) intersection of convex sets is a convex set;

b) closed balls are convex sets.

These two properties form in the considered space structure of convexity.

Several mathematicians have attempted to transfer the structure of convexity to spaces that are not vector spaces. For example, to metric spaces - W. Takahashi [29], J. P. Penot [27], W. A. Kirk [20], T. H. Kim and W. A. Kirk [18], to topological spacesM. R. Taskovič [30], C. D. Horvath [13] and to an arbitrary set (with the help of closure operators)-A. Liepiņš [24].

2000 Mathematics Subject Classification: 52A01, 47H10, 54H25.

Key words and phrases: strictly convex Banach space, nonexpansive mappings, strictly convex metric space, strictly convex metric space with round balls, normal structure, fixed point theorem.

* Née Galiņa.

The paper is in final form and no version of it will be published elsewhere. 
In this article we study strictly convex metric spaces and strictly convex metric spaces with convex round balls as a generalization of the concept of strictly convex Banach space. Some interesting results in fixed point theory have been proved in strictly convex Banach spaces, for example, M. Edelstein [9], [10], F. E. Browder [4] and R. Kannan [16] (uniformly convex Banach space is also strictly convex), Z. Opial [26], W. G. Dotson [8], K. Goebel and W. A. Kirk [12], P. Kuhfitting [23]. We prove also some fixed point theorems in strictly convex metric spaces with round balls.

2. Definitions and some properties. Now we consider the class of strictly convex spaces introduced by Clarkson and by Krein (see V. I. Istratescu [14]).

Definition 2.1. A normed linear space $X$ is said to be strictly convex (or rotund) if the boundary of its unit ball contains no line segments.

Many equivalent characterizations of strict convexity are known. For example, M. M. Day ([7], p. 111) used the following definition: the unit ball $B$ in a Banach space is rotund if every open segment in $B$ is disjoint from the boundary of $B$ (see also other properties [7], p. 112).

Let $(X, d)$ be a metric space with metric $\mathrm{d}$.

Definition 2.2 (I. Galinga [11]). A set $K \subset X$ is said to be convex if for each $x, y \in K$ and for each $t \in[0,1]$ there exists $z \in K$ that satisfies

$$
d(x, z)=t d(x, y) \quad \text { and } \quad d(z, y)=(1-t) d(x, y)
$$

and the set

$$
\{z \in K \mid d(x, z)=t d(x, y), d(z, y)=(1-t) d(x, y), t \in[0,1]\}
$$

is said to be the segment joining $x$ and $y$ (denoted $[x, y]$ ).

We note that according to this Definition 2.2 closed balls may be non-convex sets and intersection of convex sets may be a non-convex set (see, for example, I. Galiņa [11]). Therefore we define strictly convex metric spaces in following way.

Definition 2.3 (I. Galinga [11]). A metric space $(X, d)$ is said to be strictly convex if for each $x, y \in X$ and for each $t \in[0,1]$ there exists a unique $z \in X$ that satisfies

$$
d(x, z)=t d(x, y) \quad \text { and } \quad d(z, y)=(1-t) d(x, y) .
$$

This is not a new original definition; we can find it in, for example, W. Takahashi [29]. In 1992 the author of this article has proved (I. Galinga [11]) that the following conditions are equivalent in a Banach space $X$ :

1. $\forall x, y \in X:\|x+y\|=\|x\|+\|y\| \Rightarrow((\exists \lambda>0: x=\lambda y) \vee(x=0) \vee(y=0))$; 2. $\forall x, y \in X \forall t \in[0,1] \exists ! z \in X:\|x-z\|=t\|x-y\|,\|z-y\|=(1-t)\|x-y\|$.

Since the first condition is equivalent to strict convexity of a Banach space (see, for example, V. I. Istratescu [14]), we conclude that strictly convex Banach spaces indeed are strictly convex metric spaces. 
It is easy to prove that intersection of convex sets (in the sense of Definition 2.2) is a convex set in a strictly convex metric space (I. Galiņa [11]). But there exist strictly convex metric spaces in which closed balls are not convex.

ExAmPle 2.1 (I. Bula and J. Vīksna [6]). Let $X$ be an unbounded angle that is strictly less than 2 radians, with one side on the axis of polar coordinates and with vertex at the origin. We choose two arbitrary points $x=\left(r_{1}, \varphi_{1}\right)$ and $y=\left(r_{2}, \varphi_{2}\right)$ (in polar coordinates).

The distance between $x$ and $y$ is defined by

$$
d(x, y)=\left|\varphi_{1}-\varphi_{2}\right| \min \left\{r_{1}, r_{2}\right\}+\left|r_{1}-r_{2}\right| .
$$

This space $(X, d)$ is a strictly convex metric space in which closed balls are not convex.

Since we cannot guarantee that closed balls in a strictly convex metric space are convex sets, we make the following definition.

Definition 2.4 (I. Bula [5]). A strictly convex metric space $(X, d)$ is said to have round balls if

$$
\forall a, b, c \in X(a \neq b) \forall z \in[a, b](a \neq z \neq b), \quad d(c, z)<\max \{d(c, a), d(c, b)\} .
$$

It can be proved that the condition

$$
\forall a, b, c \in X(a \neq b) \forall z \in[a, b](a \neq z \neq b) \quad d(c, z) \leq \max \{d(c, a), d(c, b)\}
$$

is equivalent to convexity of closed balls. This strict inequality (2.1) shows that if $a$ and $b$ belong to the boundary of the ball $B(c, r)$ then $z$ does not belong to this sphere, i.e., a sphere does not contain straight line segments, and therefore in Definition 2.4 we speak of round balls.

We can prove one significant result

ASSERTION 2.1. Every strictly convex normed linear space $X$ is a space with round balls.

Proof. Let $a, b, c \in X, a \neq b$ and $z \in[a, b], a \neq z \neq b$. Since $X$ is strictly convex space, $\exists ! t \in] 0,1[$ such that $z=t a+(1-t) b$.

Since for every $\alpha, \beta \in \mathbf{R}, 0 \leq \alpha<\beta$ and every $t, 0<t<1$ we have $\alpha<t \alpha+(1-t) \beta<$ $\beta$, it follows that

$$
\begin{aligned}
\|c-z\| & =\|c-(t a+(1-t) b)\|=\|(t+(1-t)) c-(t a+(1-t) b)\| \\
& =\|t(c-a)+(1-t)(c-b)\| \leq t\|c-a\|+(1-t)\|c-b\| \\
& <\max \{\|c-a\|,\|c-b\|\}
\end{aligned}
$$

if $\|c-a\| \neq\|c-b\|$. If $\|c-a\|=\|c-b\|$ then from the inequalities (2.2) it follows that $\|c-z\| \leq\|c-a\|=\|c-b\|$. From $\|c-z\|=\|c-a\|=\|c-b\|$ it follows that the points $a, b, z$ belong to a sphere with center at $c$ and radius $r=\|c-z\|$, but this means that this sphere contains line segments $(z \in[a, b]$ !) - this contradicts the strict convexity of $X$. Therefore $\|c-z\|<\|c-a\|=\|c-b\|$.

We notice that well known metric spaces $\mathbf{R}$ and $\mathbf{R}^{2}$ with Euclidean metric and each of their convex subsets are strictly convex metric spaces and strictly convex metric spaces with round balls. But $\mathbf{R}^{2}$ with maximum metric or modulus metric is not a strictly convex metric space. In Example 2.1 we have shown that there exists a strictly convex metric 
space where closed balls are not convex - that is an example of a space that is not a strictly convex metric space with round balls.

The concept of normal structure has been worked out by M. Brodskij and D. Milman [3] in 1948 (in Banach spaces). This concept has been widely studied and has many variations, for example, W. Takahashi [29], J. P. Penot [27]. We use

Definition 2.5. A convex set $K$ in a metric space $X$ is said to have normal structure if each bounded convex subset $H \subset K$ with at least two points contains a nondiametral point $y \in H$, i.e.,

$$
\exists y \in H: \quad \sup \{d(x, y) \mid x \in H\}<\operatorname{diam} H=\sup \{d(x, z) \mid x, z \in H\} .
$$

We can prove the following result

LEMma 2.1 (I. Bula [5]). Every convex and bounded set in a strictly convex metric space $X$ with round balls has normal structure.

3. Fixed points. A topological space $X$ is said to have the fixed-point property if every continuous map of $X$ into $X$ has at least one fixed point. A well-known Tychonoff theorem asserts: if $X$ is a locally convex topological linear space and $K$ is a compact convex subset of $X$ then $K$ has the fixed-point property. But V. L. Klee [22] shows that any convex non-compact subset of a locally convex metrizable topological linear space lacks the fixed-point property. Fixed-point property in an arbitrary topological linear space is an important unsolved problem. We give only some positive results.

Inspired by fixed point theorems where the condition of normal structure is used we can prove

TheOREM 3.1 (I. Bula [5]). Let $(X, d)$ be a strictly convex metric space with round balls. Let $K \subset X$ be convex and compact set. If $f: K \rightarrow K$ is a nonexpansive mapping then $f$ has a fixed point in $K$.

We can prove similar theorems not only for nonexpansive mappings but also for following mappings

Definition 3.1 (R. Kannan [15], [16]). A self-mapping $f$ of a metric space $(X, d)$ is said to be a Kannan type mapping if for each pair $x, y \in X$

$$
d(f(x), f(y)) \leq \frac{1}{2}(d(x, f(x))+d(y, f(y)) .
$$

It may be noted that nonexpansiveness implies the continuity of the mapping while the Kannan type has no such implications.

If the mapping is Kannan type then $\forall x, y \in X$

$$
d(f(x), f(y)) \leq \frac{1}{2}(d(x, f(x))+d(y, f(y)) \leq \max \{d(x, f(x)), d(y, f(y))\} .
$$

We prove

TheOREM 3.2. Let $(X, d)$ be a strictly convex metric space with round balls. Let $K \subset X$ be a nonempty convex and compact set. If $f: K \rightarrow K$ is a continuous mapping and

$$
\forall x, y \in X: d(f(x), f(y)) \leq \max \{d(x, f(x)), d(y, f(y))\}
$$

then $f$ has a unique fixed point in $K$. 
Proof. From Zorn's lemma in the collection of all nonempty convex and closed subsets of $K$ each of which is mapped into itself by $f$ there exists a minimal element $K_{0}$. We show that $K_{0}$ consists of a single point. Suppose diam $K_{0}>0$.

Since $K_{0}$ is a convex and bounded set $\left(K_{0} \subset\right.$ compact set $\left.K\right)$, by Lemma $2.1 K_{0}$ has normal structure, i.e.,

$$
\exists x \in K_{0}: \sup \left\{d(x, y) \mid y \in K_{0}\right\}=r<\operatorname{diam} K_{0} .
$$

We denote the convex closed hull of the set $f\left(K_{0}\right)$ with $\overline{c o} f\left(K_{0}\right)=K_{1}$. Since $f\left(K_{0}\right) \subset$ $K_{0}$ and $f$ is continuous then

$$
K_{1}=\overline{c o} f\left(K_{0}\right) \subset \overline{c o} K_{0}=K_{0}, \quad f\left(K_{1}\right) \subset f\left(K_{0}\right) \subset \overline{c o} f\left(K_{0}\right)=K_{1} .
$$

The minimality of $K_{0}$ implies $K_{1}=K_{0}$.

Put

$$
C=\left(\bigcap_{y \in K_{0}} B(y, r)\right) \cap K_{0} .
$$

It is nonempty ( since $x \in C$ ), convex (by Lemma 2.1 closed balls are convex sets) and closed (as the intersection of closed sets).

We define

$$
C_{1}=\left(\bigcap_{y \in f\left(K_{0}\right)} B(y, r)\right) \cap K_{0} .
$$

Since $f\left(K_{0}\right) \subset K_{0}$ then $C_{1} \supset C$. If $z \in C_{1}$ then

$$
f\left(K_{0}\right) \subset B(z, r) \text { and } K_{0}=K_{1}=\overline{c o} f\left(K_{0}\right) \subset B(z, r)
$$

(because $B(z, r)$ is a closed and convex set) therefore $C \supset C_{1}$. It follows that $C=C_{1}$.

Now we define

$$
M=\left(\bigcap_{y \in f(C)} B(y, r)\right) \cap C .
$$

It is nonempty since $x \in C$ and $\sup \left\{d(x, y) \mid y \in K_{0}\right\}=r$, i.e., $x \in B(y, r), \forall y \in f(C)$, that is convex and closed set as intersection of convex and closed sets.

We choose freely $z \in M \subset C$ and $y \in f(C)$. Then exists $w \in C$ such that $y=f(w)$. Thereby

$$
d(y, f(z))=d(f(w), f(z)) \leq \max \{(d(w, f(w)), d(z, f(z))\} .
$$

Since $w, z \in C \subset K_{0}$ then $f(w), f(z) \in K_{0}$ and therefore $d(w, f(w)) \leq r, d(z, f(z)) \leq r$ by definition of $C$. Therefore $d(f(z), y) \leq r$ for any $y \in f(C)$, i.e., $f(z) \in M$ or $f: M \rightarrow$ $M$. The minimality of $K_{0}$ implies $M=K_{0}$. But

$$
\operatorname{diam} M \leq r<\operatorname{diam} K_{0} .
$$

From the obtained contradiction we conclude that diam $K_{0}=0$ and $K_{0}=\left\{x^{*}\right\}$ and therefore $f\left(x^{*}\right)=x^{*}$.

The uniqueness of the fixed point follows from condition (3.1).

Corollary 3.1. Let $(X, d)$ be a strictly convex metric space with round balls. Let $K \subset X$ be a convex and compact set. If $f: K \rightarrow K$ is a continuous mapping with (3.1) and $F=\left\{f_{i} \mid f_{0}=f, f_{i}: K \rightarrow K, i=1,2, \ldots\right\}$ is a commutative family of mappings then there exists a unique common fixed point for the family $F$. 
Proof. By Theorem 3.2 the mapping $f$ has a unique fixed point $x^{*} \in K$. This point is a common fixed point for the family $F$. Since

$$
\forall f_{i} \in F: \quad f_{i}\left(x^{*}\right)=f_{i}\left(f\left(x^{*}\right)\right)=f\left(f_{i}\left(x^{*}\right)\right), \quad i=1,2, \ldots,
$$

then $f_{i}\left(x^{*}\right), i=1,2, \ldots$, are fixed points for $f$. By uniqueness it follows that $f_{i}\left(x^{*}\right)=x^{*}$, $i=0,1,2, \ldots$.

Remarks. 1. A simple example shows that if the conditions of Theorem 3.2 hold but $f$ is not continuous then it may lack a fixed point.

We fix $x_{0}, y_{0} \in K, x_{0} \neq y_{0}$. The mapping $f: K \rightarrow K$ for every $x \in K$ is defined by

$$
f(x)= \begin{cases}x_{0}, & x \neq x_{0} \\ y_{0}, & x=x_{0}\end{cases}
$$

Then $f$ satisfies (3.1) (but is not Kannan type): since $\forall x, y \in K\left(x \neq x_{0}, y \neq x_{0}\right)$ $d(f(x), f(y))=0$ and if $x=x_{0}$ then

$$
d\left(f\left(x_{0}\right), f(y)\right)=d\left(y_{0}, x_{0}\right) \leq \max \left\{d\left(x_{0}, f\left(x_{0}\right)\right), d(y, f(y))\right\}=\max \left\{d\left(x_{0}, y_{0}\right), d\left(y, x_{0}\right)\right\} .
$$

2. Let $M=\left\{a_{1}, a_{2}, a_{3}\right\}$, and define the metric in $M$

$$
d\left(a_{i}, a_{j}\right)= \begin{cases}0, & i=j \\ 1, & i \neq j .\end{cases}
$$

The set $M$ is compact but not convex. The mapping $f: M \rightarrow M$ defined as $f\left(a_{1}\right)=a_{2}$, $f\left(a_{2}\right)=a_{3}, f\left(a_{3}\right)=a_{1}$ is continuous and satisfies condition (3.1) but lacks a fixed point.

3 . Let $B$ denote the unit ball in the Hilbert space $l_{2}$ with norm $\|x\|=\sqrt{\sum_{i=1}^{\infty} x_{i}^{2}}<$ $\infty$. This space, being a uniformly convex space, is a strictly convex space (also with round balls), the unit ball is convex but weakly compact. In this situation there exists a continuous mapping without fixed point. See for example W. A. Kirk [19].

4. Examples showing that strict convexity and round balls are necessary conditions in Theorem 3.2 are not easy to find. Obviously these examples are connected with the unsolved problem of existence of fixed points for a continuous mapping in a convex and compact set in an arbitrary space. Theorem 3.2 gives only sufficient conditions for the solution of this problem.

\section{References}

[1] L. Blumenthal, Theory and Applications of Distance Geometry, Oxford, 1953.

[2] T. Botts, Convex sets, Amer. Math. Monthly 49 (1942), 527-535.

[3] M. S. Brodskij and D. P. Milman, On the center of a convex set, Dokl. Akd. Nauk SSSR (N.S.) 59 (1948), 837-840.

[4] F. E. Browder, Nonexpansive nonlinear operators in a Banach space, Proc. Nat. Acad. Sci. USA 54 (1965), 1041-1044.

[5] I. Bula, Strictly convex metric spaces and fixed points, Mathematica Moravica 3 (1999), $5-16$.

[6] I. Bula and J. Vīksna, Example of strictly convex metric space with not convex balls, submitted. 
[7] M. M. Day, Normed Linear Spaces, second corrected printing, Ergeb. Math. Grenzgeb. 21, Springer, 1962.

[8] W. G. Dotson, Fixed points of quasi-nonexpansive mappings, J. Austral. Math. Soc. 13 (1972), 167-170.

[9] M. Edelstein, On non-expansive mappings of Banach spaces, Proc. Camb. Phil. Soc. 60 (1964), 439-447.

[10] M. Edelstein, Fixed point theorems in uniformly convex Banach spaces, Proc. Amer. Math. Soc. 44 (1974), 369-374.

[11] I. Galiņa, On strict convexity, LU Zinātniskie Raksti, Matemātika 576 (1992), 193-198.

[12] K. Goebel and W. A. Kirk, A fixed point theorem for asymptotically nonexpansive mappings, Proc. Amer. Math. Soc. 35 (1972), 171-174.

[13] C. D. Horvath, Contractibility and Generalized Convexity, J. Math. Analysis and Applications 156 (1991), 341-357.

[14] V. I. Istratescu, Fixed Point Theory: an Introduction, Math. and its Applications, 1981.

[15] R. Kannan, Some results on fixed points - II, Amer. Math. Monthly 76 (1969), 405-408.

[16] R. Kannan, Fixed point theorems in reflexive Banach space, Proc. Amer. Math. Soc. 38 (1973), 111-118.

[17] D. C. Kay and E. W. Womble, Axiomatic convexity theory and relationships between the Carathéodory, Helly and Radon numbers, Pacific J. Math. 38 (1971), 471-485.

[18] T. H. Kim and W. A. Kirk, Convexity and metric fixed point theory, in: Nonlinear Analysis in Geometry and Topology, Th. Rassias (ed.), Hadronic Press, Palm Harbor, FL, 2000, $107-124$.

[19] W. A. Kirk, A fixed point theorem for mappings which do not increase distances, Amer. Math. Monthly 72 (1965), 1004-1006.

[20] W. A. Kirk, Fixed point theory for nonexpansive mappings II, Contemporary Math. 18 (1983), 121-140.

[21] V. L. Klee, Convex sets in linear spaces, Duke Math. J. 18 (1951) 443-466.

[22] V. L. Klee, Some topological properties of convex sets, Trans. Amer. Math. Soc. 78 (1955), $30-45$.

[23] P. Kuhfitting, Fixed points of several classes of nonlinear mappings in Banach space, Proc. Amer. Math. Soc. 44 (1974), 300-306.

[24] A. Liepiņš, A cradle-song for a little tiger on fixed points, Topological Spaces and their Mappings, Riga, 1983, 61-69.

[25] K. Menger, Untersuchungen über allgemeine Metrik, Math. Ann. 100 (1928), 75-163.

[26] Z. Opial, Weak convergence of the sequence of successive approximations for nonexpansive mappings, Bull. Amer. Math. Soc. 73 (1967), 591-597.

[27] J. P. Penot, Fixed point theorems without convexity, Bull. Soc. Math. France 60 (1979), 129-152.

[28] V. P. Soltan, Introduction to the Axiomatic Theory of Convexivity, Kishinev (Moldova), 1984 (in Russian).

[29] W. Takahashi, A convexity in metric space and nonexpansive mappings I, Kōdai Math. Semin. Rep. 22 (1970), 142-149.

[30] M. R. Taskovič, General convex topological spaces and fixed points, Math. Moravica 1 (1997), 127-134. 